\title{
Measuring Method for the Interference Contrast of Multi-Beam-Interference
}

\author{
$\underline{\text { Michael STEGER }}^{{ }^{*}, 2}$, Simon BOES $^{* 2}$, Sven THILKER $^{{ }^{*}}$, Arnold GILLNER $^{{ }^{*} 1,2}$ \\ ${ }^{* 1}$ Chair for Laser Technology, Technical University Aachen, Steinbachstr. 15, \\ 52074 Aachen, Germany \\ ${ }^{* 2}$ Fraunhofer Institute for Lasertechnology, Steinbachstr. 15, 52074 Aachen, Germany \\ E-Mail: michael.steger@ilt.fraunhofer.de
}

\begin{abstract}
Multi-Beam-Interference (MBI) is a promising approach for the direct nano structuring of surfaces. An important characteristic of MBI is the interference contrast which describes the modulation depth of the intensity distribution. While theoretical calculation for the interference contrast exists in literature, no actual measurement in experiments is documented. In this paper we present a method for determining the interference contrast by measuring the virtual shift of the ablation threshold. This virtual shift is caused by the modified intensity distribution via interference. At first, the theoretical basis for the measuring method is introduced, secondly the method is tested with a two beam interference setup used for direct structuring of surfaces. The measuring method shows consistent results and proves to be a useful tool for characterization of the interference pattern.

DOI: $10.2961 / \mathrm{jlmn} .2014 .03 .0008$
\end{abstract}

Keywords: Multi-beam interference, interference contrast, nano structuring, polyimid

\section{Introduction}

The functionalization of surfaces is a key technology for many applications. The wide variety of functions for these applications ranges from wettability and tribology to optical properties [1-3]. The challenge in generating the desired function is not only the feasibility of the structuring process, but also the costs of this process. Lithographic processes are commonly used for the fabrication of nano structures. They allow the reproduction of complex and freely defined structures, but are very cost-intensive due to many complex process steps and high-precision photomasks. For a broad integration of surface functionalization in consumer products a significant decrease of costs is necessary.

Multi-Beam Interference (MBI) is a promising approach to decrease the structuring costs of periodic surface functionalizations [2]. It allows the direct nano structuring of surfaces without the use of expensive masks and generates periodic structures simultaneously over an area. The size of the area depends on the beam diameter and the ablation threshold of the material. With the use of ultra short pulse laser a wide variation of materials can be processed.

An important characteristic for the description of MBI is the interference contrast $[4,5]$. The interference contrast describes the quality of the intensity modulation via the difference between the maxima and minima of the intensity distribution. Currently MBI is mostly used in the context of lithographic purposes as means to avoid expensive photomasks [2]. For lithographic patterning one main constraint exists: the intensity maxima have to be above the crosslinking threshold of the photoresist and the minima below. The intensity used for the exposure of the resist is relatively low and is homogeneously distributed over the whole processed area. Therefore the contrast of the interference pattern is of secondary concern compared to the uniformity of the pattern.
For direct surface structuring by MBI the contrast is of much greater importance. The intensity maxima of the interference modulation are directly linked to the ablation depth, while the intensity minima define the amount of energy deposited in the surface area where no alteration is desired. In case of a reduced contrast more energy is deposited at the minima leading to unwanted heating, ablation or even destruction of the whole pattern. A low contrast leads to a shift of the intensity distribution from the ablation area to the area where no alterations are wanted. Therefore if a certain depth of the pattern is targeted a higher total intensity is needed in case of a low contrast. Thus, the efficiency of the structuring process is significantly reduced. The ablation threshold for the direct nano structuring of materials is much higher than the reaction threshold of photoresists. This means that the percentage of the total process cost for the laser source is much higher compared to lithographic processes. In order to achieve good results and a costefficient production a high contrast needs to be used.

While theoretical calculation and optimization of the interference contrast for different beam setups and restraints exists in literature [4,5], no publication indicates the actual interference contrast achieved during direct ablation. A direct measurement of the interference pattern is not possible due to the resolution limit of sensors. The aim of this paper is to present an indirect method for measurement of the interference contrast by a footprint approach. To verify this method a test with a two-beam interference setup is carried out and compared to theoretically expected values.

\section{Measuring the interference contrast}

The interference pattern from MBI is created at the intersection of two or more coherent laser beams. These patterns are periodic and their periodicity $\Lambda$ depends on the incidence angle $\alpha$ of the intercepting beams and the laser wavelength with $\Lambda=\lambda /(2 \sin (\alpha))$ for a two-beam inter- 
ference. This intensity modulation, which is caused by interference, occurs in the whole volume where the beams intercept as depicted in figure 1.

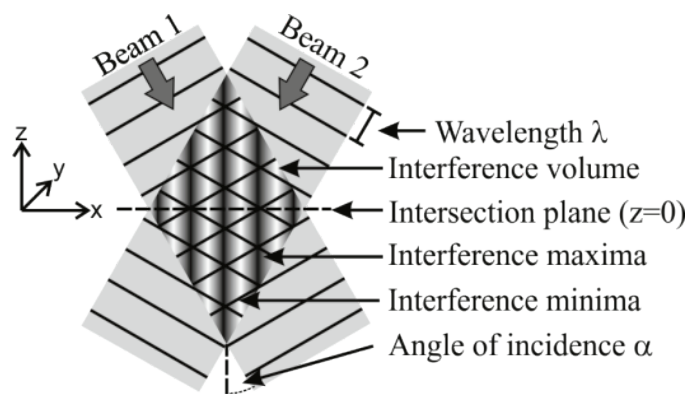

Figure 1: Schematic diagram of an interference volume of two intercepting beams with a wavelength $\lambda$ and an angle of incidence $\alpha$.

The total time independent intensity distribution $I(\boldsymbol{r})$ at the intersection of the number of $\mathrm{j}$ plane waves is given by [6] :

$$
\begin{gathered}
I(\boldsymbol{r})=\sum_{j} I_{j}(\boldsymbol{r})+2 \sum_{i<j} \sqrt{I_{i}(\boldsymbol{r}) I_{j}(\boldsymbol{r})} \cos \left(\theta_{i j}\right) \\
* \cos \left(\left(\mathbf{K}_{i}-\boldsymbol{K}_{j}\right) \boldsymbol{r}+\phi_{i}-\phi_{j}\right)
\end{gathered}
$$

Where $I_{j}$ is the intensity of beam $\mathrm{j}, \theta_{i j}$ is the angle between the unit vectors of beam $i$ and $j$ in its polarization direction, $\boldsymbol{K}_{\boldsymbol{j}}$ is the wave vector of beam $\mathrm{j}$ and $\phi_{i}$ the phase of beam $\mathrm{i}$.

The first sum of equation 1 describes the total intensity $I_{0}$ without interference. The second sum defines the modulation around $I_{0}$ due to the interference. Depending on the ratio of the intensity $I_{j}$ of each wave and the angle $\theta_{i j}$ the modulation depth, which equals the interference contrast, ranges from 0 to $I_{0}$. The general interference contrast is defined as:

$$
\text { Contrast }=\frac{I_{\max }-I_{\min }}{I_{\max }+I_{\min }}
$$

For a two beam interference of identical Gaussian beams equation (1) can be written as:

$$
\begin{gathered}
I(\boldsymbol{r})=\left[I_{1}+I_{2}+2 \sqrt{I_{1} I_{2}} \cos \left(\theta_{12}\right)\right. \\
\left.* \cos \left(\left(\mathbf{K}_{1}-\boldsymbol{K}_{2}\right) \boldsymbol{r}+\phi_{i}-\phi_{j}\right)\right] e^{\left(-4 r^{2} / \omega^{2}\right)}
\end{gathered}
$$

For $I_{1}+I_{2}=I_{0}$ the envelope of the intensity maxima of the modulation can be simplified from equation (3) to:

$$
I_{\text {int }}=I_{0} e^{\left(-4 r^{2} / \omega^{2}\right)}(1+k)
$$

Where $\omega$ is the beam radius and $\mathrm{k}$ is defined as interference contrast $k=\cos \left(\theta_{i j}\right)$ ranging from 0 to 1 . The increase in peak intensity is therefore linked to the interference contrast.

Although this example is shown for two-beam interference, this approach can be applied to any MBI. Figure 2 shows an example for a two beam interference with a Gaussian intensity distribution for an ideal contrast of 1 and a reduced contrast of 0.25 . The dashed lines in the graphs show the envelope of the intensity maxima of equation (4).
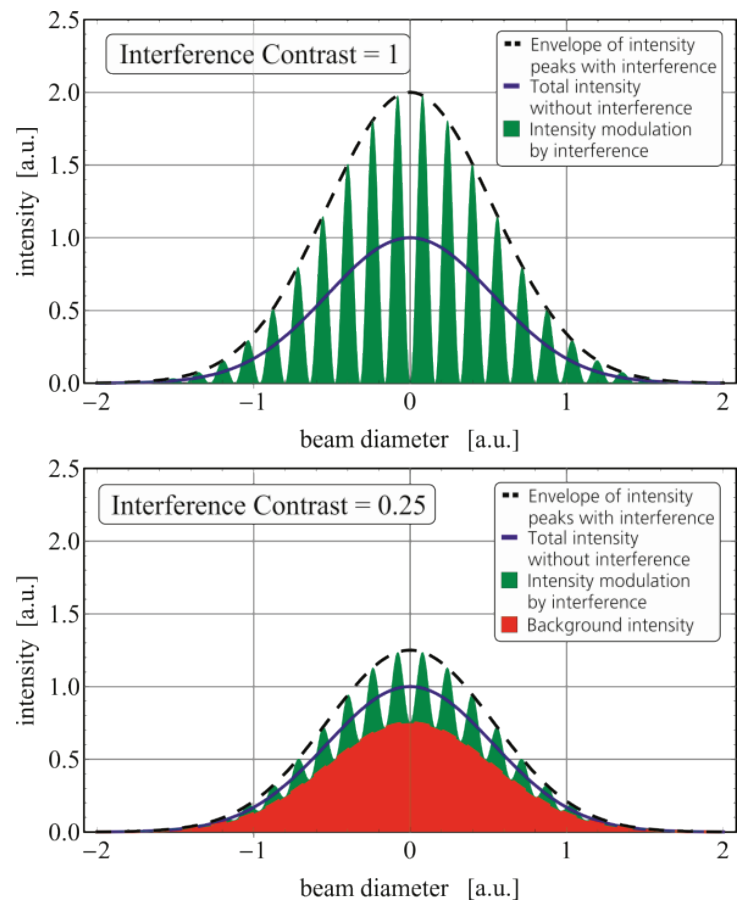

Figure 2: Intensity profiles of a two-beam interference with a Gaussian intensity distribution. The upper image shows an interference contrast of 1 and in the lower image an interference contrast of 0.25 . The total intensity $\boldsymbol{I}_{\mathbf{0}}$ is identical in both cases.

If MBI interference is used to structure a surface, the higher the contrast is, the earlier the ablation threshold is surpassed at a lower total intensity $I_{0}$ or at a larger diameter for a fixed total intensity $I_{0}$ due to the higher peak intensities of the interference modulation. This redistribution of the intensity lead for interference to larger structured diameter which is only patterned and not completely ablated as for a non-modulated beam. This correlation is illustrated in figure 3 .

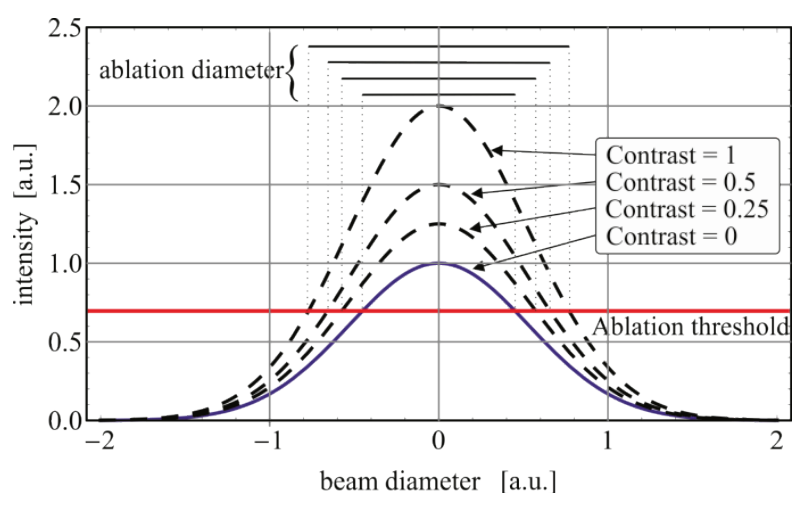

Figure 3: Intensity profiles for the envelope of the peak intensities of the interference pattern. For a fixed total intensity $\boldsymbol{I}_{\mathbf{0}}$ the diameter of the area, where ablation occurs, increases in correlation with the interference contrast.

Measuring the ablation threshold of a material for MBI with a total intensity $I_{0}$, the observed ablation threshold differs depending on the interference contrast $\mathrm{k}$. This virtual shift of the ablation threshold allows the determination 
of the interference contrast. For a Gaussian intensity distribution the interference contrast can be calculated through equation (4).

The value of the interference contrast depends on the intensity distribution of each beam and their ability to interfere with each other. The ability to interfere depends on the angle $\theta_{i j}$ between the E-vectors of both beams and also on the coherence of the beams. Due to the limited coherence length of a laser source, a deviation in the path length of the partial beams decreases their ability to interfere. Therefore an alteration of $\mathrm{k}$ is necessary:

$$
k=\cos \left(\theta_{i j}\right) k_{c o h}
$$

Where $k_{c o h}$ is a factor for the coherence of beams which describes the ability of the beams to interfere if a temporal shift between them is present. For no temporal shift the factor is 1 , while for an increasing shift it decreases towards 0 . This point is reached when the temporal shift caused by deviation in path length is greater than the coherence length of the laser.

\section{Experimental setup and method}

To verify the presented measuring method of the interference contrast, the results were tested via a two-beam interference setup. The setup is shown in figure 4 :

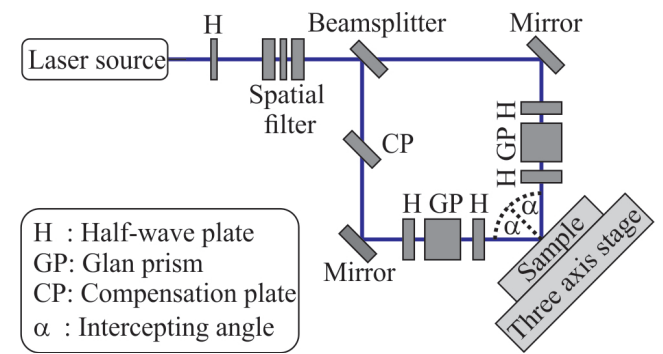

Figure 4: Schematic diagram of the two-beam interference setup

The laser source is a diode pumped Nd:YAG laser (Q301-HD, JDSU) which operates at a wavelength of $355 \mathrm{~nm}$ with a pulse duration of $38 \mathrm{~ns}$. The coherence length is $5.4 \mathrm{~mm}$ calculated by the spectral width of the emitted beam by the Wiener-Chintschin-Theorem. The emitted beam is turned to a perpendicular polarization by a half-wave plate in relation to the plane of the optical table. Afterwards the beam is refined through a spatial room filter and splitted into two by a $50 \%$ reflective beam splitter. Directed by mirrors, the partial beams superpose at the sample surface. For an equal beam path of both beams a compensation plate of same material as the beam splitter is added into the reflected beam path. The angle $\alpha$ can be adjusted and it controls the periodicity of the interference pattern. The sample material is polyimid foil (Upilex) with a thickness of $50 \mu \mathrm{m}$. For further control of the beam parameters an optional system of two half-wave plates and a Glan prism can be integrated into the partial beam paths to adjust the polarization and the intensity of each beam separately.

The output of the laser is measured by an energy detector (QE12LP-S-MP, Gentec) and a beam profiler (Spiricon, Ophir) at the intersection of the beams. A typical beam diameter is $\sim 900 \mu \mathrm{m}$ with maximum pulse energy of $1.2 \mathrm{~mJ}$. The samples are structured by a single pulse. The maxi- mum diameter of the ablated area is about $600 \mu \mathrm{m}$. For ablation without interference the structuring is carried out with one beam being blocked and with both beams for MBI. The diameter of the ablated area is measured by a Laser-Scanning Microscope (VK-9700, Keyence).

\section{Experimental results}

To allow a comparison between the theoretically expected and the measured value for the interference contrast, it is necessary to determine the experimental parameters which define the contrast as stated in equation (1).

The total intensity ratio between the beams is nearly identical with a deviation of $3 \%$. The intensity distribution of the single beams and the superposed beams is shown in figure 5 .

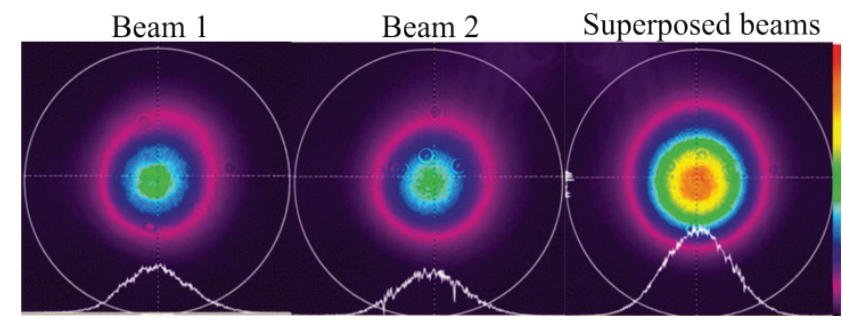

Figure 5: Measurement of the beam shape for the partial beams and the superposed beams in the intersection plane.

All three profiles exhibit a Gaussian intensity distribution with a goodness of fit of over 0.98 . For the superposition of both beams the measured diameter increases by $1.8 \%$. This minimal increase shows that the positioning of the beams is nearly ideal in the sample plane i.e. the $x-y$ plane. The sample surface is positioned in the center of the interference volume as shown in figure 1 by tracking the gap between the beams when moving the sample in $\mathrm{z}$ direction. Thus the values of the intensity $I_{1}$ and $I_{2}$ for both beams are almost identical at every point in the sample plane resulting in a good alignment of the beams.

The ability of the beams to interfere depends on the angle of their polarization and the coherence of the beams to each other. The polarization of the beams is chosen perpendicular to the plane of incidence, so the angle $\theta_{i j}$ is 0 and independent from the angle of incidence $\alpha$. The optical path length of beams for the temporal coherence is adjusted by checking the generated structures on a sample surface in order to achieve an optimal coherence. If a beam path is changed in length relative to the other one, the nano structures on the sample surface diminish and finally vanish due to temporal incoherence.

For the above stated parameters the theoretical value of the interference contrast is close to 1 assuming the sample is positioned in the center of the interference volume and that the coherence of both beams is ideal.

In the next step polyimide foil is structured by MBI with four different periodicities by changing the intersection angle of the two beams. This is repeated without interference by blocking one beam. The four periodicities are 560, 635, 790 and $1000 \mathrm{~nm}$. For each periodicity the pulse intensity is varied from below the ablation threshold to the maximal intensity of the laser source and the radii of the ablated spots are measured. By plotting the global maximum peak intensity of each pulse versus the radii of the 
ablation areas the ablation thresholds can be extrapolated by applying a logarithmic fit [7]. The ablation threshold can is identified by the intersection point between the extrapolated logarithmic fit and longitudinal $\mathrm{x}$-axis. A plot of the experimental results is shown in figure 7 for different periodicities of the interference pattern.

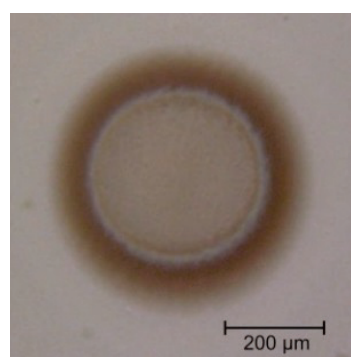

Figure 6: Example for the irradiated sample surface. The periodicity of the interference pattern is $1 \mu \mathrm{m}$ with a peak fluence 0.3 $\mathrm{J} / \mathrm{cm}^{2}$ for the Gaussian shaped laser beam.

The ablation threshold for structuring without interference is between 121 and $125 \mathrm{~mJ} / \mathrm{cm}^{2}$ which is in good agreement with literature values [8]. Higher values occur for smaller periodicities. This behavior is to be expected due to the increasing angle of incidence leading to an increase in reflection at the sample surface. The ablation threshold for structuring with interference is lower than without interference and ranges from 81 to $89 \mathrm{~mJ} / \mathrm{cm}^{2}$.

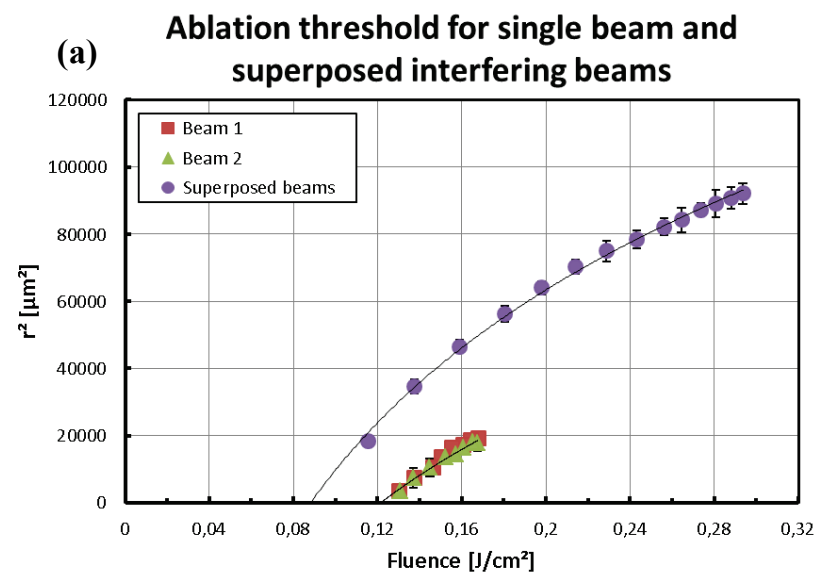

(b)

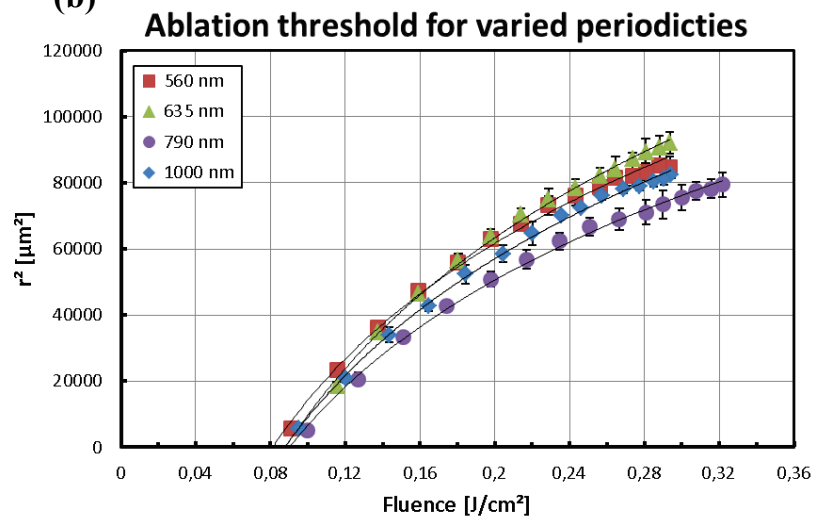

Figure 7: Plot of the peak intensity of a single beam or the superposed beams versus the square of the radii of the ablated zones on a polyimid sample. The upper graph (a) depicts the shift due to interference relative to the threshold without interference. The lower graph (b) shows the behavior of this shift in relation to the periodicity of the interference pattern
Derived from equation (4) with $F_{\text {single }}$ and $F_{\text {interference }}$ the thresholds for single beam and interference ablation:

$$
F_{\text {single }}(1+0)=F_{\text {interference }}(1+k)
$$

The interference contrast $\mathrm{k}$ can be calculated by dividing the measured ablation thresholds:

$$
\frac{F_{\text {single }}}{F_{\text {interference }}}-1=k
$$

In table 1 the interference contrasts calculated via equation (6) are listed.

Table 1 Interference contrast derived from comparison of ablation thresholds with and without interference

\begin{tabular}{c|cc|c}
\hline $\begin{array}{c}\text { Period } \\
{[\mathrm{nm}]}\end{array}$ & \multicolumn{2}{|c|}{ Threshold $\left[\mathrm{mJ} / \mathrm{cm}^{2}\right]$} & $\begin{array}{c}\text { Interference } \\
\text { Contrast }\end{array}$ \\
\hline 560 & 125 & MBI & 81 \\
635 & 121 & 88 & 0.54 \\
790 & 123 & 89 & 0.38 \\
1000 & 121 & 87 & 0.38 \\
\hline
\end{tabular}

The measured interference contrast ranges from 0.38 to 0.54 with no clear influence of the periodicity of the interference pattern. Compared to the theoretically expected interference contrast close to 1 , the measured value is approximately divided by two.

In a second experiment the polarization of one beam is varied relative to the polarization of the other beam from $0^{\circ}, 60^{\circ}$ and $90^{\circ}$. In case of $0^{\circ}$ the experimental conditions are similar to the previous experiments with added optical components for controlling the polarization of both beams separately. For $60^{\circ}$ the expected interference contrast should be halved and for $90^{\circ}$ the contrast is expected to be 0 as given by equation (1). The varied angle $\theta_{i j}$ between the polarization vectors of each beam decrease their ability to interfere by $\cos \left(\theta_{i j}\right)$ as shown in the equation. The results of the experiment are shown in figure 8 .

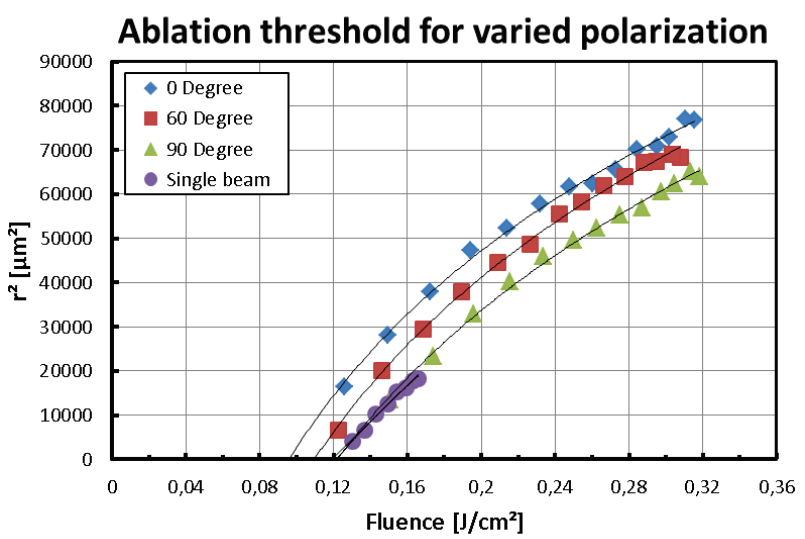

Figure 8: Plot of the peak intensity of the superposed beams versus the square of the radii of the ablated zones for different angles between the unit vectors of both beams in their polarization direction. 
The ablation thresholds for $0^{\circ}, 60^{\circ}, 90^{\circ}$ are 95,109 , $122 \mathrm{~mJ} / \mathrm{cm}^{2}$. The calculated values for the interference contrast, which are derived from the relation of the shifted ablation threshold to the single beam ablation, are $0.26,0.11$ and 0 . The measured values show a good fit to the theoretically expected values. The interference contrast for $0^{\circ}$ is further decreased compared to the previous experiments due to introduction of more optical components into the partial beam paths. To summarize the results from the experiments:

- A shift of the ablations thresholds due to interference is measurable.

- The estimated values for the interference contrast from the ablation threshold are in the correct range of 0 to 1 .

- The measured interference contrast for different periodicities ranges from 0.39 to 0.54 which is lower than expected.

- A reduction of the interference contrast by varying the polarization decreases the contrast as theoretically expected.

Does the measured interference contrast match the real contrast during the experiment or is there a deviation due to the measuring method?

The experimental results indicate that the measured interference contrast is reasonable for the following reasons: The data points from the laser fluence versus the radii of the ablated spots fit well to a logarithmic curve for different periodicities, which shows that the results are consistent for different intensities and periodicities of the interference pattern. Furthermore the observed effect of an intentional decrease of interference contrast by modification of the polarization is as theoretically expected.

If the interference contrast is correctly measured, the parameters on which the theoretical calculations are based must be different than expected. While the beam shape, intensity and polarization can be precisely measured, the method for adjusting the path lengths of both beam for ideal coherence give no exact result for $k_{c o h}$ and can lead to suboptimal conditions for the interference contrast. If the previous assumption of good coherence with $k_{c o h}$ close to 1 is not correct, the reached interference contrast is significantly reduced by this factor as equation (5) states.

\section{Conclusion}

The interference contrast is an important characteristic for the interference pattern applied by MBI. It describes the modulation depth of the pattern and is therefore closely linked to the minimal size and aspect ratio of the generated structures by direct laser ablation. A direct measurement of the interference contrast is not possible due to insufficient resolution of sensors. To circumvent this limitation this publication presents the theoretical basis for measuring the interference contrast by a footprint approach and experimentally tests this method for a two-beam interference. The measurement is based on the variation of the peak intensity depending on the interference contrast at a constant total intensity. The height of the intensity peaks are coupled to the interference contrast. To observe the higher peak intensity the diameter of structured areas on polyimide foil were measured in an experiment. Due to the increased peak intensity a virtual shift of the ablation threshold compared to the threshold of non-modulated beam was observed. The size of shift allows the determination of the interference contrast. The measured values for the interference contrast show consistent results. Whether the measured values equal the actual interference contrast cannot be determined with absolute certainty, although there is a strong indication for a correct measurement. Even if the measured value does not equal the actual one, there exists a proportional link between the two. Therefore the presented method is a useful tool for measurement and optimization of structuring by MBI.

\section{Acknowledgments}

Parts of the here depicted research was funded by the German Research Foundation (DFG) as part of the Cluster of Excellence "Integrative Production Technology for HighWage Countries" and as part of the DFG priority program "Generation of sub-100 nm structures by nonlinear lasermaterial interactions".

\section{References}

[1] SH. Baik, SK. Hwang, YG. Kim, GJ. Park, JH. Kwon, WT. Moon, SH. Kim, BK. Kim, SH. Kang: Journal of the Optical Society of Korea, 13, (2009), pp. 478-483

[2] G. M. Burrow, T.K. Gaylord: Micromachines 2011, Vol. 2, 221-257

[3] D. Langheinrich et al: Journal of Polymer Science Part B: Polymer Physics, 50, (2012), pp. 415-422

[4] L.Z. Cai, X.L. Yang and Y.R. Wang: Journal of Modern optics, 49, (2002), pp. 1663-167

[5] G. M. Burrow, T.K. Gaylord: Journal Of MicroNanolithography Mems And Moems, Vol. 11, Issue 4

[6] E. Hecht: “Optik" (Oldenbourg, Munich, 2005) p.624

[7] J. M. Liu: Optics Letters, Vol. 7, No. 5, May 1982

[8] S. Küper, J. Brannon, and K. Brannon: Appl. Phys. A56, 43-50 (1993)

(Received: March 31, 2014, Accepted: August 27, 2014) 